Claremont Colleges

Scholarship@ Claremont

All HMC Faculty Publications and Research

HMC Faculty Scholarship

$12-1-1997$

\title{
On Multiple Solutions of a Nonlinear Dirichlet Problem
}

\author{
Alfonso Castro \\ Harvey Mudd College \\ Jorge Cossio \\ Universidad Nacional de Colombia \\ John M. Neuberger \\ Mississippi State University
}

\section{Recommended Citation}

Castro, Alfonso, Jorge Cossio and John M. Neuberger. "On multiple solutions of a nonlinear Dirichlet problem”, Nonlinear Analysis TMA, Vol.30, No. 6 (1997), pp. 3657-3662.

This Article - postprint is brought to you for free and open access by the HMC Faculty Scholarship at Scholarship @ Claremont. It has been accepted for inclusion in All HMC Faculty Publications and Research by an authorized administrator of Scholarship @ Claremont. For more information, please contact scholarship@cuc.claremont.edu. 
PII: S0362-546X(96)00240-4

\title{
ON MULTIPLE SOLUTIONS OF A NONLINEAR DIRICHLET PROBLEM
}

\author{
ALFONSO CASTRO ${ }^{1}$, JORGE COSSIO $^{2}$, and JOHN M. NEUBERGER ${ }^{3}$ \\ ${ }^{1}$ Department of Mathematics, University of North Texas, \\ Denton, Texas 76203-5116, USA; \\ ${ }^{2}$ Departamento de Matemáticas, Universidad Nacional de Colombia, \\ Apartado Aéreo 3840, Medellín, Colombia; and \\ ${ }^{3}$ Department of Mathematics, Mississippi State University, \\ Mississippi State, MS 39762, USA.
}

\begin{abstract}
We prove that a semilinear elliptic boundary value problem has five solutions when the range of the derivative of the nonlinearity includes at least the first two eigenvalues. We also prove that if the region is a ball the semilinear elliptic problem has two solutions that change sign and are nonradial.
\end{abstract}

Key words and phrases: Nonlinear Dirichlet problem, multiplicity of solutions.

\section{INTRODUCTION}

Let $f: \mathbb{R} \rightarrow \mathbb{R}$ be a differentiable function such that $f(0)=0$, and

$$
f^{\prime}(\infty)=\lim _{|u| \rightarrow \infty} \frac{f(u)}{u} \in \mathbb{R} .
$$

Let $\Omega$ be a smooth bounded region in $\mathbb{R}^{n}$, and $\Delta$ the Laplacian operator. Let $\lambda_{1}<\lambda_{2} \leq \cdots \leq$ $\lambda_{k} \leq \ldots$ be the eigenvalues of $-\Delta$ with homogeneous Dirichlet boundary conditions in $\Omega$. The solvability of the boundary value problem

$$
\left\{\begin{aligned}
\Delta u+f(u)=0 & \text { in } \Omega \\
u=0 & \text { on } \partial \Omega
\end{aligned}\right.
$$

has proven to be closely related to the position of the numbers $f^{\prime}(0), f^{\prime}(\infty)$ with respect to the spectrum of $-\Delta$. In fact, A. Castro and A. Lazer in [9] showed that if the interval $\left(f^{\prime}(0), f^{\prime}(\infty)\right) \cup\left(f^{\prime}(\infty), f^{\prime}(0)\right)$ contains the eigenvalues $\lambda_{k}, \ldots, \lambda_{j}$ and $f^{\prime}(t)<\lambda_{j+1}$ for all $t \in \mathbb{R}$, then (1.2) has at least three solutions. The proofs in [9] are based on global LyapunovSchmidt arguments applied to variational problems. Subsequently K.C. Chang (see [10]) approached the same problems using Morse theory, and H. Hofer (see [12]) obtained the existence of five solutions when $f^{\prime}(\infty)<\lambda_{1}$. For other results in the study of this problem we refer the reader to [2], [3], [5], [6], [7], [13], [14], and [15], among others.

This research was partially supported by NSF grant DMS-8905936 and Colciencias grant 168-93 
Our first result concerns the existence of multiple solutions of (1.2).

THEOREM A. If $f^{\prime}(0)<\lambda_{1}, f^{\prime}(\infty) \in\left(\lambda_{k}, \lambda_{k+1}\right)$ with $k \geq 2$, and $f^{\prime}(t) \leq \gamma<\lambda_{k+1}$ for all $t \in \mathbb{R}$, then (1.2) has at least five solutions. Moreover, one of the following cases occur:

a) $k$ is even and (1.2) has two solutions that change sign.

b) $k$ is even and (1.2) has six solutions, three of which are of the same sign.

c) $k$ is odd and (1.2) has two solutions that change sign.

d) $k$ is odd and (1.2) has three solutions of the same sign.

The assumption $k \geq 2$ is sharp; Theorem B of [9] gives sufficient conditions for (1.2) to have exactly three solutions when $k=1$. We prove Theorem A by using Lyapunov-Schmidt arguments to reduce the solvability of (1.2) to a finite dimensional problem, and then we use degree and index theories applied to critical points. We also use "mountain pass arguments" of the type Ambrosetti-Rabinowitz (see [4]).

Next we state a result for (1.2) where $\Omega$ is the unit ball in $\mathbb{R}^{n}$ centered at the origin, and $\lambda_{1}=\lambda_{1}^{r}<\lambda_{2}^{r}<\cdots<\lambda_{k}^{r}<\ldots$ are the eigenvalues of $-\Delta$ acting on radial functions of $H_{0}^{1}(\Omega)$.

THEOREM B. If $f^{\prime}(0)<\lambda_{1}, f^{\prime}(\infty) \in\left(\lambda_{k}, \lambda_{k+1}\right)$ with $k \geq 2, f^{\prime}(t) \leq \gamma<\lambda_{k+1}$ for all $t \in \mathbb{R}$, $\lambda_{1}<\lambda_{k}<\lambda_{k+1} \leq \lambda_{2}^{r}$, and $t f^{\prime \prime}(t)>0 \quad \forall t \neq 0$, then the boundary value problem (1.2) has at least two solutions which are nonradial and change sign.

In Section 2 we sketch the proof of Theorem A, the details of the proof can be found in [8]. Theorem B is new, and its proof will be given in Section 3.

\section{SKETCH OF PROOF OF THEOREM A}

Let $H$ be the Sobolev space $H_{0}^{1}(\Omega)$. We say that $u \in H$ is a weak solution of (1.2) if for every $\varphi \in H$

$$
\int_{\Omega}(\nabla u . \nabla \varphi-f(u) \varphi) d x=0
$$

By standard regularity for elliptic operators (see [9]) it follows that weak solutions are classical solutions when $f$ is continuous and sublinear.

Let $J: H \rightarrow \mathbb{R}$ denote the functional defined by

$$
J(u)=\int_{\Omega}\left(\frac{1}{2}\|\nabla u\|^{2}-F(u)\right) d x
$$

wherc $F(\xi)=\int_{0}^{\xi} f(s) d s$. Since $f^{\prime}(\infty) \in\left(\lambda_{k}, \lambda_{k+1}\right), f$ is sublinear. Thus $J \in C^{1}(H, \mathbb{R})$ (see [15]) and

$$
\langle\nabla J(u), \varphi\rangle=\int_{\Omega}(\nabla u \cdot \nabla \varphi-f(u) \varphi) d x \quad \text { for } \quad \varphi \in H .
$$

In particular $u$ is a weak solution of (1.2) if and only if $u$ is a critical point of $J$. Since $f(0)=0$, it follows that $u^{0} \equiv 0$ is a trivial solution of $(1.2)$. Since 0 is an isolated local minimum of $J$ we have

$$
d\left(\nabla J, B_{0}, 0\right)=1
$$


where $B_{0}$ is a ball centered at zero containing no other critical point (see [1]).

Using mountain pass arguments, and degree theory we prove the following lemma.

Lemma 2.1 (see [8]). Under the hypotheses of Theorem A, (1.2) posseses a positive solution $u^{+}$and a negative solution $u^{-}$. If the set of positive (respectively, negative) solutions is discrete then at least one of them is a critical point of mountain-pass type and its local degree is -1 . If $P$ is a bounded region containing the positive solutions to (1.2) and no other critical point of $J$ then

$$
d(\nabla J, P, 0)=-1
$$

If $N$ is a bounded region containing the negative solutions to (1.2) and no other critical point of $J$ then

$$
d(\nabla J, N, 0)=-1
$$

By the Lyapunov-Schmidt reduction process we now prove the following lemma.

Lemma 2.2. There exists $u_{0} \in H$ such that $\nabla J\left(u_{0}\right)=0$, and if $u_{0}$ is an isolated critical point then

$$
d(\nabla J, V, 0)=(-1)^{k}
$$

in any region $V$ containing $u_{0}$ and no other critical point of $J$.

Proof. For each positive integer $n$ let $\varphi_{n}$ denote an eigenfunction corresponding to the eigenvalue $\lambda_{n}$. Let $X$ denote the subspace of $H$ spanned by $\left\{\varphi_{1}, \varphi_{2}, \ldots, \varphi_{k}\right\}, Y$ its orthogonal complement, and $J$ the functional defined by (2.1). By Lemma 2.1 of [8] it follows that there exists a continuous function $\psi: X \rightarrow Y$ such that

$$
J(x+\psi(x))=\min _{y \in Y} J(x+y) .
$$

Moreover, $\psi(x)$ is the unique member of $Y$ such that

$$
\langle\nabla J(x+\psi(x)), y\rangle=0 \quad \text { for all } \quad y \in Y,
$$

the function $\tilde{J}: X \rightarrow \mathbb{R}$ defined by $\tilde{J}(x)=J(x+\psi(x))$ is of class $C^{1}$, and

$$
\left\langle\nabla \tilde{J}(x), x_{1}\right\rangle-\left\langle\nabla J(x+\psi(x)), x_{1}\right\rangle \quad \text { for all } \quad x, x_{1} \in X .
$$

We now claim that for $x \in X$

$$
J(x) \rightarrow-\infty \quad \text { as } \quad\|x\| \rightarrow \infty .
$$

Because $f^{\prime}(\infty) \in\left(\lambda_{k}, \lambda_{k+1}\right)$ there exist $b \in \mathbb{R}$ and $\bar{\gamma}>\lambda_{k}$ such that $F(\xi) \geq \frac{\bar{\gamma} \xi^{2}}{2}+b$. Hence

$$
J(x)=\frac{1}{2}\|x\|^{2}-\int_{\Omega} F(x) \leq \frac{1}{2}\|x\|^{2}-\frac{\bar{\gamma}}{2} \int_{\Omega} x^{2}-b|\Omega|
$$


Since $\langle x, x\rangle \leq \lambda_{k}\langle x, x\rangle_{0}$ for $x \in X$, where $\langle., .\rangle_{0}$ denotes the usual inner product in $L^{2}(\Omega)$, we obtain

$$
J(x) \leq \frac{1}{2}\|x\|^{2}\left(1-\frac{\bar{\gamma}}{\lambda_{k}}\right)-b|\Omega| \longrightarrow-\infty \quad \text { as } \quad\|x\| \rightarrow \infty .
$$

Because $\dot{J}(x) \leq J(x)$, we have

$$
\tilde{J}(x) \rightarrow-\infty \quad \text { as } \quad\|x\| \rightarrow \infty .
$$

Since $\operatorname{dim} X<\infty$ there exists $x_{0} \in X$ such that

$$
\tilde{J}\left(x_{0}\right)=\max _{x \in X} J(x+\psi(x)) .
$$

Taking $u_{0}=x_{0}+\psi\left(x_{0}\right)$ we have $\nabla J\left(u_{0}\right)=0$. Suppose now that $x_{0}$ is an isolated critical point of $j$, hence $u_{0}$ is an isolated critical point of $J$. Since $-j$ has a local minimum at $x_{0}$, taking $W=\{x \in X ; x+\psi(x) \in V\}$ then $d(\nabla \tilde{J}, W, 0)=(-1)^{k}$. Therefore (see Lemma 2.1 of [8]) we have

$$
d(\nabla J, V, 0)=(-1)^{k}
$$

and Lemma 2.2 has been proved.

Suppose $k$ is even, and $K$, the set of critical points of $J$, is finite. By Lemma 2.1 we have

$$
d(\nabla J, P, 0)=d(\nabla J, N, 0)=-1,
$$

where $P$ is a bounded region containing the positive solutions to (1.2) and no other critical point of $J$, and $N$ is a bounded region containing the negative solutions to (1.2) and no other critical point of $J$.

Since $f^{\prime}(\infty)$ is not an eigenvalue of $-\Delta$ with zero Dirichlet boundary conditions, the solutions of (1.2) are bounded. Because the Leray-Schauder degree of the gradient of a coercive functional on a large ball is one (see [1]), we have $d\left(\nabla \tilde{J}, B_{R}, 0\right)=(-1)^{k}=1$, for a large enough value of $R$.

If $u_{0} \notin P \cup N$ then by the invariance of the Leray-Schauder degree under the LyapunovSchmidt reduction method (see [8]), and by the excision and existence properties of the Leray-Schauder degree there exists $u_{1} \notin\left(\overline{P \cup N \cup B_{0} \cup V}\right)$ such that $\nabla J\left(u_{1}\right)=0$, which proves that (1.2) has at lcast five solutions. In this case both $u_{0}$ and $u_{1}$ change sign.

Suppose now that $u_{0} \in P \cup N$; without loss of generality we can assume that $u_{0} \in P$. By Lemma 2.1 there exists a critical point of mountain-pass type $u_{1} \in P$ and its local degree is -1 . By the excision property of the Leray-Schauder degree there exists another solution $u_{2} \in P$. Finally by the excision and the existence properties of the Leray-Schauder degrce there exists $u_{3} \notin\left(P \cup N \cup B_{0}\right)$ with $\nabla J\left(u_{3}\right)=0$. Which shows that (1.2) has six solutions. In this case $u_{0}, u_{1}, u_{2}$ have the same sign. This completes the proof of Theorem $\mathrm{A}$ when $k$ is even. Similar arguments prove Theorem A when $k$ is odd.

\section{PROOF OF THEOREM B}

Since the nonlinearity $f$ satisfies the hypotheses of Theorem A, it follows that problem (1.2) has at least five solutions. By Lemma 2.1 it follows that there exist a positive solution $u^{+}$ 
and a negative solution $u^{-}$to (1.2). By the so-called moving planes Theorem of [11], it follows that $u^{+}$and $u^{-}$are radially symmetric. We now prove that there are exactly two nonzero radially symmetric solutions to (1.2). We recall that radial solutions to (1.2) are solutions to the ordinary differential equation

$$
\left\{\begin{aligned}
u^{\prime \prime}+\frac{n-1}{r} u^{\prime}+f(u) & =0 \quad(0<r \leq 1) \\
u(1)=u^{\prime}(0) & =0 .
\end{aligned}\right.
$$

First, we show that the radial positive solution to problem (1.2) is unique. Let $v_{1}>0$ and $v_{2}>0$ be radial solutions to (1.2) and $v=v_{1}-v_{2}$. Therefore, $v$ satisfies

$$
\left\{\begin{aligned}
v^{\prime \prime}+\frac{n-1}{r} v^{\prime}+\frac{f\left(v_{1}\right)-f\left(v_{2}\right)}{v_{1}-v_{2}} v & =0 \quad(0<r \leq 1) \\
v(1)=v^{\prime}(0) & =0 .
\end{aligned}\right.
$$

From the mean value theorem, we have

$$
\left\{\begin{aligned}
v^{\prime \prime}+\frac{n-1}{r} v^{\prime}+f^{\prime}(\xi) v & =0 \\
v(1)=v^{\prime}(0) & -0,
\end{aligned} \quad(0<r \leq 1)\right.
$$

where $\xi \in\left(v_{1}, v_{2}\right)$. Let $\varphi_{2}$ be an eigenfunction corresponding to $\lambda_{2}^{r}$. One sees that $\varphi_{2}$ satisfies

$$
\varphi_{2}^{\prime \prime}+\frac{n-1}{r} \varphi_{2}^{\prime}+\lambda_{2}^{r} \varphi_{2}=0 .
$$

We now show that $v$ does not change sign. If we suppose that it does, since $f^{\prime}(\xi) \leq \gamma<\lambda_{2}^{r}$ then by the Sturm comparison 'I'heorem (see [16]) we see that $\varphi_{2}$ has at least 2 zeroes in $[0,1)$. This is a contradiction. Without loss of generality we may assume that $v_{1} \geq v_{2}$. Also, since $f$ is convex we have

$$
\frac{f\left(v_{1}\right)}{v_{1}} \geq \frac{f\left(v_{2}\right)}{v_{2}}
$$

Note that $v_{1}$ and $v_{2}$ satisfy

$$
\begin{aligned}
& v_{1}^{\prime \prime}+\frac{n-1}{r} v_{1}^{\prime}+\frac{f\left(v_{1}\right)}{v_{1}} v_{1}=0, \\
& v_{2}^{\prime \prime}+\frac{n-1}{r} v_{2}^{\prime}+\frac{f\left(v_{2}\right)}{v_{2}} v_{2}=0 .
\end{aligned}
$$

Hence, if $v_{1} \neq v_{2}$, say $v_{1}(x)>v_{2}(x)$ for some $x \in[0,1)$, then again by the Sturm comparison Theorem we see that $v_{1}$ has at least one zero in $[0,1)$. This contradicts that $v_{1}>0$, thus we have shown that problem (1.2) has a unique positive solution. Similar arguments prove that (1.2) has a unique negative solution.

We now suppose that $v$ is a radially symmetric solution to (1.2) which changes sign. Therefore, $v$ satisfies

$$
\left\{\begin{aligned}
v^{\prime \prime}+\frac{n-1}{r} v^{\prime}+\frac{f(v)}{v} v & =0 & (0<r \leq 1) \\
v^{\prime}(0)=v(1) & =0 . &
\end{aligned}\right.
$$

By our hypothesis and the mean value theorem, we see that $\frac{f(v)}{v}<\lambda_{2}^{r}$. Thus, comparing (3.2) with (3.3), we see that $\varphi_{2}$ has at least 2 zeroes in $[0,1)$. This is also a contradiction, 
and so it follows that if $v$ is a radially symmetric solution to (1.2) then $v$ does not change sign.

We have shown that the problem (1.2) has exactly two nonzero radially symmetric solutions. Since $u^{0} \equiv 0$ is a solution and the problem (1.2) has at least five solutions, the remaining two solutions are nonradial and change sign. This proves Theorem B.

\section{REFERENCES}

1. H. AMMAN, A note on degree theory for gradient mappings, Proc. Amer. Math. Soc. 85 (1982), 591-595.

2. H. AMMAN and E. ZEHNDER, Nontrivial solutions for a class of nonresonance problems and applications to nonlinear differential equations, Ann. Scuola Norm. Sup. Pisa 7 (1980), 539-603.

3. A. AMBROSETTI and G. MANCINI, Sharp nonuniqueness results for some nonlinear problems, Nonlinear Anal, theory Methods 3 (1979), 635-645.

4. A. AMBROSETTI and P. RABINOWITZ, Dual variational methods in critical point theory, J. Funct. Anal. 14 (1973), 343-381.

5. L. BOCCARDO and T. GALLOUËT, Homogenization for "Castro-Lazer Equation, Nonlinear Anal. 14 (1990), 81-91.

6. N. P. CÁC, On nontrivial solutions of an asymptotically linear Dirichlet problem, J. Differential Equations 75 (1988), 103-107.

7. A. CASTRO, Métodos variacionales y análisis funcional no lineal, X Coloquio Colombiano de Matemáticas, Paipa, Colombia, 1980.

8. A. CASTRO and J. COSSIO, Multiple solutions for a nonlinear Dirichlet problem, SIAM J. Math. Anal. 25, No. 6 (1994), 1554-1561.

9. A. CASTRO and A. C. LAZER, Critical point theory and the number of solutions of a nonlinear Dirichlet problem, Ann. Mat. Pura Appl. (4) 120 (1979), 113-137.

10. K. C. CHANG, Solutions of asymptotically linear operator equations via Morse theory, Comm. Pure Appl. Math. 34 (1981), 693-712.

11. B. GIDAS, W. NI and L. NIRENBERG, Symmetry and related properties via the maximum principle, Comm. Math. Phys. 68 (1979), 209-243..

12. H. HOFER, Variational and topological methods in partially ordered Hilbert spaces, Math. Ann. 261 (1982), 493-514.

13. A. C. LAZER and S. SOLIMINI, Nontrivial solutions of operator equations and Morse indices of critical points of min-max type, Nonlinear A nal. 12 (1988), 761-775.

14. S. LI and J. Q. LIU, Nontrivial critical points for asymptotically quadratic function, Report of International Center for Theorethical Physics IC/390, Trieste, Italy., 1986.

15. P. RABINOWITZ, Minimax methods in critical point theory with applications to differential equations, Regional conference series in Mathematics, number 65, AMS, Providence, Rhode Island, 1986.

16. J. SOTOMAYOR, Liçóes de equaçóes diferenciais or linárias, Instituto de Matemática Pura e Aplicada, IMPA, Rio de Janeiro, 1979. 